\title{
Wafer bonding of gallium arsenide on sapphire
}

\author{
P. Kopperschmidt, G. Kästner, St. Senz, D. Hesse, U. Gösele \\ Max-Planck-Institute of Microstructure Physics, Weinberg 2, D-06120 Halle, Germany \\ (Fax: +49-345/5511223, E-mail: kopper@ mpi-halle.mpg.de)
}

Received: 10 February 1997/Accepted: 17 February 1997

\begin{abstract}
Three-inch (100) gallium arsenide wafers were bonded to (1102) sapphire in a micro-cleanroom at room temperature under hydrophilic or hydrophobic surface conditions. Subsequent heating up to $500^{\circ} \mathrm{C}$ increased the bond energy of the GaAs-on-sapphire (GOS) wafer pair close to the fracture energy of the bulk material. The bond energy was measured as a function of the temperature. Since the thermal expansion coefficients of GaAs and sapphire are close to each other, the bonded wafer pair is stable against thermal treatment and quenching in liquid nitrogen. During heating in different gas atmospheres, macroscopic interface bubbles and microscopic imperfections were formed within the bonding interface, which were analysed by transmission electron microscopy (TEM). These interface bubbles can be prevented by hydrophobic bonding in a hydrogen atmosphere.
\end{abstract}

PACS: $68.35 ; 81.40 ; 85.30$

Direct wafer bonding (DWB) refers to the adhesion of mirrorpolished and flat surfaces of wafers of various materials to each other at room temperature. Its main application area has been silicon wafer bonding for silicon-on-insulator (SOI) materials fabrication, power devices, and silicon based sensors and actuators [1-5]. Roomtemperature bonding occurs because of attractive Van der Waals forces or hydrogen bridge bonds between a few monolayers of water molecules on the surface of the wafers. The resulting bonding energy is typically weak compared to bond energies of covalently bonded materials. Therefore, after wafer bonding at room temperature, heat treatment at elevated temperatures is required to increase the bonding energy across the bonding interface. DWB can also be demonstrated for a variety of other materials besides silicon, such as $\mathrm{Si} /$ sapphire, $\mathrm{Si} / \mathrm{GaAs}$, and other combinations [6-9]. In contrast to heteroepitaxy, DWB works independently of the crystal structure, lattice mismatch, and even crystallinity of the materials to be bonded and allows combinations of materials without the introduction of dislocations due to a difference in the lattice constants. A disadvantage of the wafer bonding approach to the combination of different materials is the presence of different thermal expansion coefficients. Thermally induced mechanical stress may cause cracking and debonding of the bonded wafers in the heat treatment required to increase the bonding energy. A few material combinations of technological interest exist for which this problem is negligible or at least tolerable, for example silicon carbide/silicon [10], germanium/sapphire, and gallium arsenide/sapphire. This article deals with DWB of gallium arsenide on sapphire.

Packaging of gallium arsenide and sapphire is of technological interest for producing integrated high-frequency devices, e.g. those based on GaAs amplifiers and microwave filters made from high-temperature superconductor films epitaxially grown on (1120)-oriented sapphire substrates [11], which offer very low dielectric loss [12]. Such devices operating at liquid nitrogen temperatures may find applications in the field of satellite and cellular phone communications. Because of the different lattice constants of (1102) sapphire and (100) GaAs it is not possible to grow GaAs of high quality on sapphire. Hence the DWB of GaAs-on-sapphire, optionally with subsequent thinning of the GaAs wafer, offers an attractive way to fabricate GaAs layers on sapphire. In this paper, we will deal with only the issue of wafer bonding.

\section{Experimental procedures and results}

Three-inch wafers of (100) GaAs and (1102) sapphire with thicknesses ranging from 400 to $500 \mu \mathrm{m}$ are positioned, in a micro-cleanroom setup, with their polished sides face to face [13]. The wafers are separated by three small spacers. The versatility of the DWB technique, means it is not necessary to take into account the relative crystallographic orientation of the wafers. Usually, the wafers are placed with their flats lining up. The mean roughness of the surfaces is lower than $0.5 \mathrm{~nm}$, measured with atomic force microscopy Fig. 1 . The wafers have a negligibly low waviness. A jet of purified water between the two wafers removes dust particles from the surfaces. Then, the micro-cleanroom is closed, and the wafers are dried by being rotated at 3300 r.p.m. for 5-7 minutes. During the rotation, the wafers are illuminated by an IR lamp, which heats the wafers to nearly $100^{\circ} \mathrm{C}$ to speed up the drying process. After this final cleaning procedure, the surfaces remain 


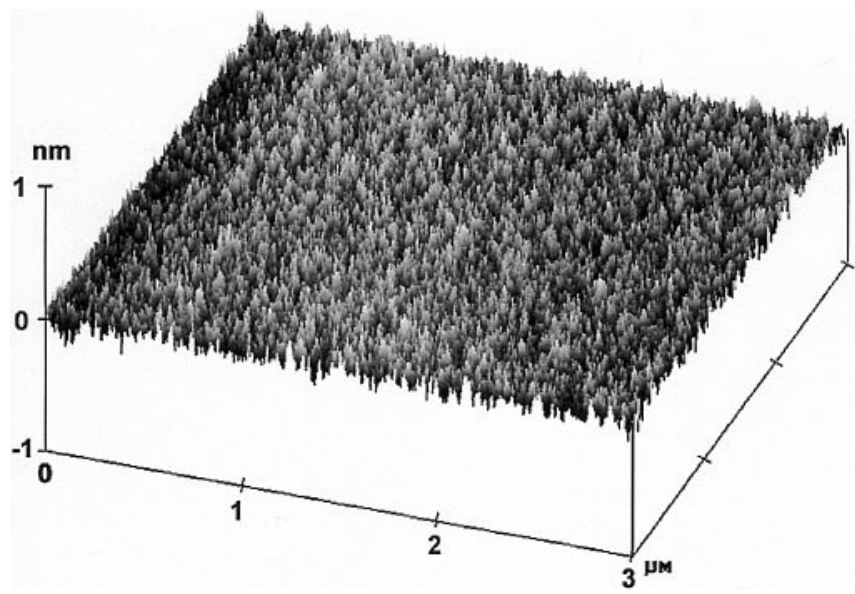

Fig. 1. GaAs surface morphology, measured by atomic force microscopy. The mean roughness is lower than $0.5 \mathrm{~nm}$, suitable for DWB

covered by a few monolayers of water molecules and hydroxyl groups and are thus hydrophilic. With the micro-cleanroom at roomtemperature and three spacers simultaneously removed and the remaining closed, the upper wafer drops onto the wafer below. Usually, bonding does not start spontaneously, but must rather be activated by local pressure, exerted by a tong. After initiation a bond front spreads over the whole surface within a few seconds. The energy per area required to separate the two bonded wafers (the so-called "bonding energy" or, more accurately, "interface energy"), measured by the crackopening method [14], varies between 250 and $400 \mathrm{~mJ} / \mathrm{m}^{2}$, These values indicate hydrogen and Van der Waals bonds and are too small for any applications.

Surface inhomogenities or particles may lead to unbonded areas, voids, or bubbles within the interface of the bonded GaAs-on-sapphire (GOS) wafer pair. Large voids can be im-

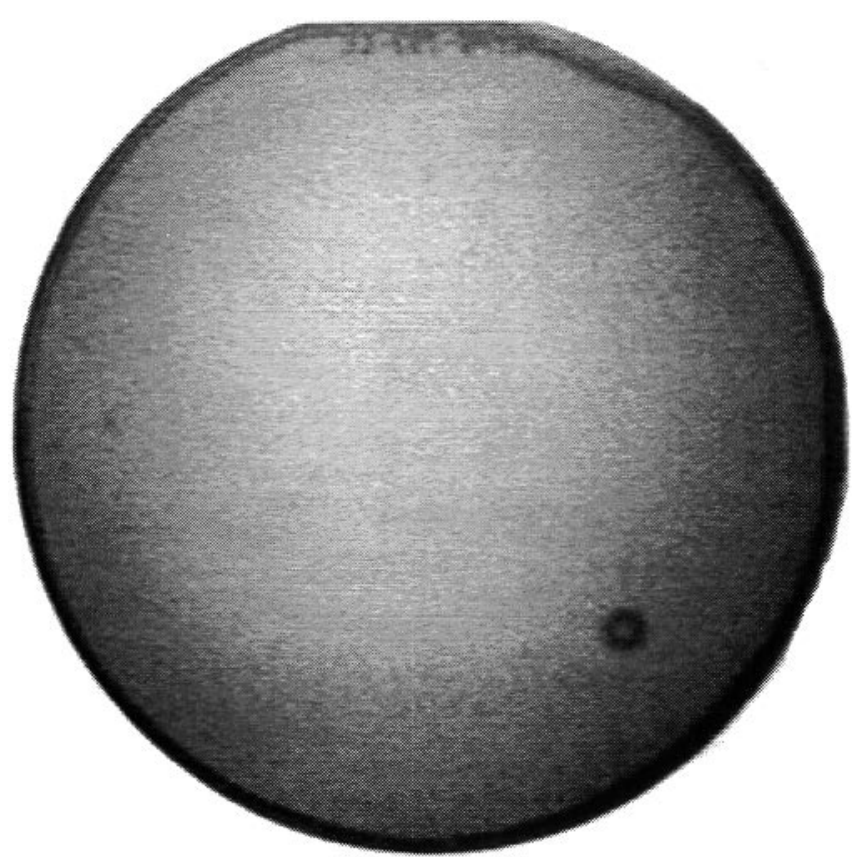

Fig. 2. Transmission infrared picture of a 3-in. GaAs wafer directly bonded on sapphire (GOS) taken at room temperature some minutes after bonding. The wafer pair is completely bonded with the exception of a small void

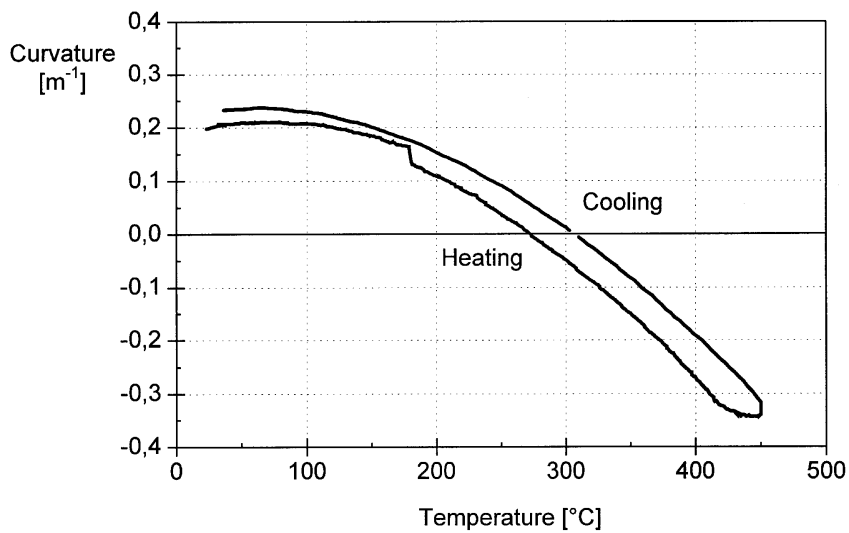

Fig. 3. Thermally induced curvature of the GOS wafer pair during heating to $500{ }^{\circ} \mathrm{C}$. The heating and cooling process is reversible

aged by transmission infrared light as shown in Fig. 2. The wafer pair is well bonded, except for a small region close to the rim of the wafer where the surface curvature caused by polishing is too strong. A dark ring indicates the existence of a void in the interface. Mechanical debonding of the wafers and repeating the cleaning and bonding procedure often reduces the occurrence of these voids.

In order to increase the bonding energy, the bonded GOS wafer pairs are heated at several hundred degrees Celsius. This thermal treatment leads to stronger chemical bonds, eliminating micro-roughness and providing more area of contact. An upper temperature limit in the heat treatment is mostly given by thermal strain if the thermal expansion coefficients of the bonded wafers differ too much. This restriction is, however, very weak in the case of GaAs-on-sapphire. The thermal expansion coefficients of GaAs and sapphire in the range of the applied temperatures are $5.8 \times 10^{-6} / \mathrm{K}$ and $5.5 \times 10^{-6} / \mathrm{K}$, respectively. The bending of the bonded GOS wafers due to the remaining difference in thermal expansion coefficients was measured by the deviation of a reflected laser beam as a function of temperature. The results are shown in Fig. 3 where the bending due to thermal strain is seen to be sufficiently low to avoid breakage or debonding. Moreover, the bonded and annealed 3-inck. GOS wafer pair withstands a dip in liquid nitrogen without breaking.

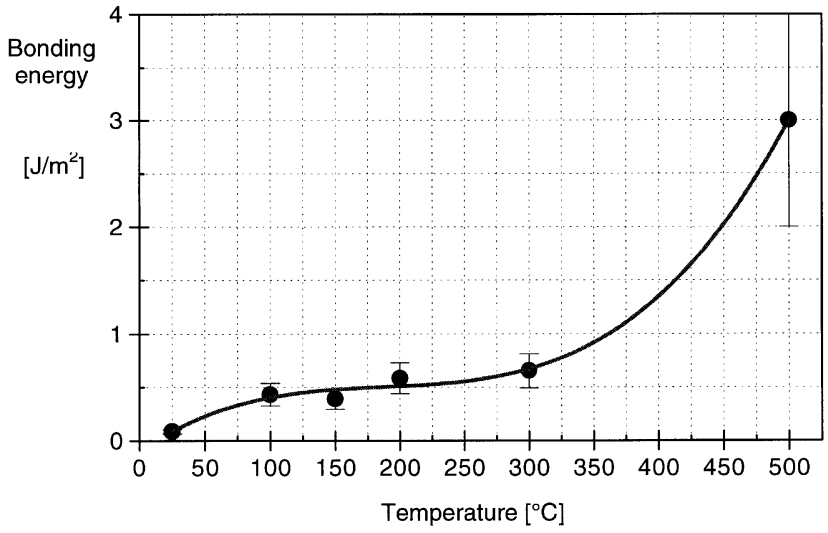

Fig. 4. Bonding energy of the GOS wafer pair as a function of temperature for successive heating 
The bonding energy of the GOS wafer pair as a function of the heating temperature is shown in Fig. 4. At low temperatures this energy presumably increases by reorganisation of the hydrogen bridges and Van der Waals bonds. Above $350{ }^{\circ} \mathrm{C}$ these bonds will increasingly be replaced by covalent bonds. High bonding energies are archieved already at relatively low temperatures, compared with the case of silicon-silicon bonding. The bonding energy obtained at $500^{\circ} \mathrm{C}$ corresponds to that of bulk GaAs. Further heating at higher temperatures has not been performed because of the associated evaporation of arsenic if this is performed in an ambient atmosphere.

The problem of degassing becomes evident by the presence of interface bubbles that are detected on a macroscopic and on a microscopic scale if the wafers are joined by hydrophilic wafer bonding. Figure 5 shows a sequence of optical in-situ photographs with growing bubbles during heating of the bonded wafer pair. As a remarkable feature, the interface bubbles, seen by the interference fringes, do not join but appear to repel each other. As the wafers are returned to room temperature the interference fringes shrink, indicating a reduced pressure inside the bubbles (see Fig. 5e). However, the interface bubbles persist even after the wafers are dipped in liquid nitrogen. We conclude that the voids initially collect some gases, surface contaminants, or oxides resulting from the dissociation of water molecules, which remain within the bubbles, thus locally preventing subsequent rebonding. In the case of silicon-silicon wafer bonding the nucleation of interface bubbles has been shown to be associated with the desorption of volatile hydrocarbon adsorbates on the surface [15]. Such voids can be avoided by a proper pretreatment of the wafer sur-
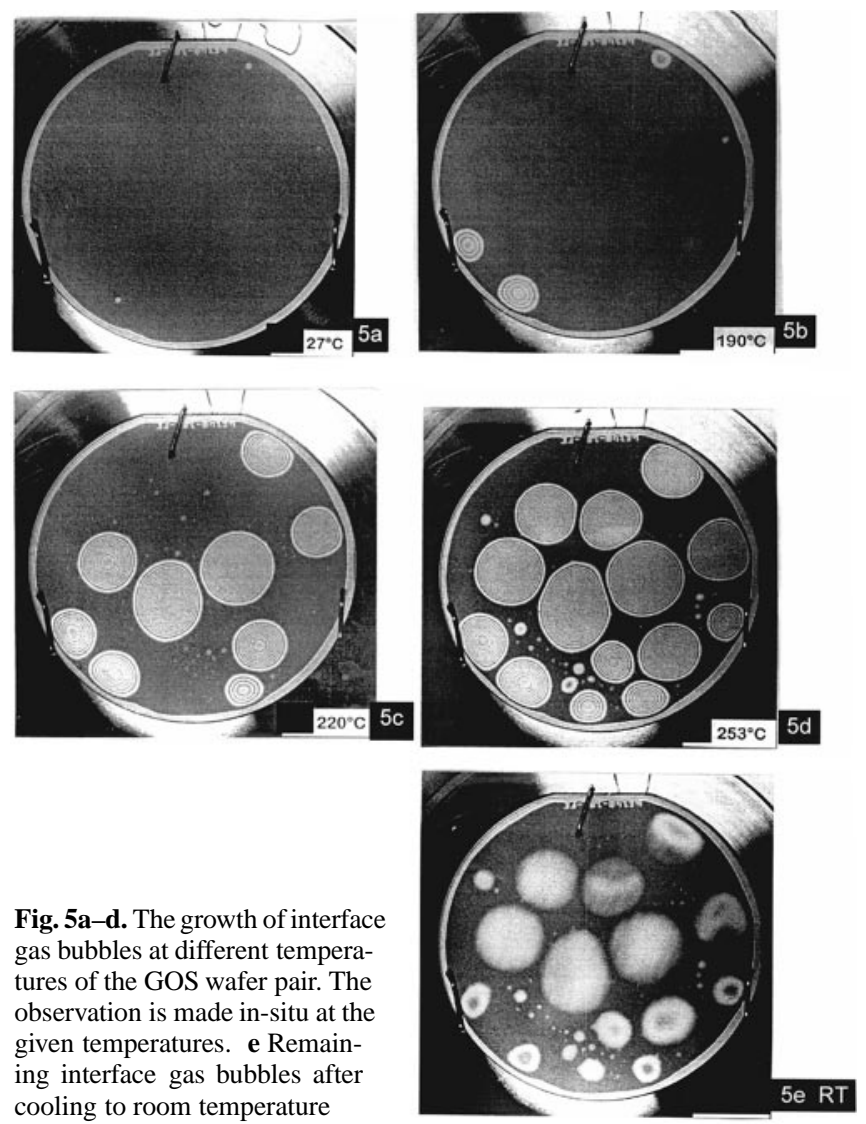

Fig. 5a-d. The growth of interface gas bubbles at different temperatures of the GOS wafer pair. The observation is made in-situ at the given temperatures. e Remaining interface gas bubbles afte cooling to room temperature faces, as will be discussed below. Substitution of hydrophilic surfaces by hydrophobic ones and subsequent bonding and annealing in hydrogen has been found to be effective for the bubble-free GOS wafer bonding.

Information on the structure and morphology of the GOS interface is obtained by transmission electron microscopy (TEM). Small pieces of the bonded area are cut by a precision saw, ground, polished, and ion-beam thinned. A $400 \mathrm{keV}$ transmission electron microscope is used for lattice imaging. A corresponding cross section is shown in Fig. 6. The transition of the GaAs lattice to that of sapphire is very sharp within a few atomic layers. Grooves or microbubbles of amorphous material with a depth of 3-10 nm and a width of $10-20 \mathrm{~nm}$ are observed within the GaAs at the interface (Fig. 7). These are identified via electron energy-loss spectroscopy and energy dispersive X-ray microanalysis as gallium-rich and arsenicrich precipitates [16]. These grooves or microbubbles might result from water-induced oxidation of GaAs. The edges of the grooves are close to the crystallographic [111] and [100] planes. It is known that the amourphous native oxides $\mathrm{Ga}_{2} \mathrm{O}_{3}$ and $\mathrm{As}_{2} \mathrm{O}_{3}$ tend to form islands of polycrystalline $\mathrm{Ga}_{2} \mathrm{O}_{3}$ in GaAs during annealing above $350^{\circ} \mathrm{C}$ [17]. Further dissociation of the remaining water molecules might be responsible for filling the grooves.

Plan-view TEM specimens - where the transmission is perpendicular to the plane of bonding - were prepared in order to check the lateral distribution of possible defects. Usually a Moiré pattern can be seen as a result of the superposition of the two different crystal lattices and their slight remaining rotational misorientation. In addition, numerous small inhomogenities with diameters below $50 \mathrm{~nm}$, separated by a mean distance of about $500 \mathrm{~nm}$, are frequently observed. They probably correspond to the grooves visible in cross sections such as Fig. 7. More rarely, a transition to larger voids is found as shown in Fig. 8. This figure reveals bonded areas that appear

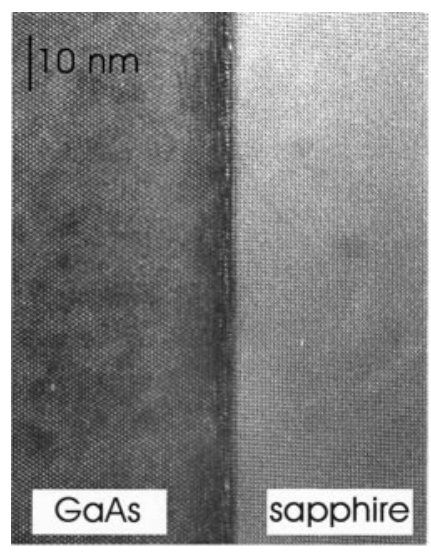

Fig. 6. High-resolution cross section TEM micrograph of the GOS interface

Fig. 7. Cross section TEM picture of a ditch filled with amorphous material. The edges of the grooves are

crystallographically oriented

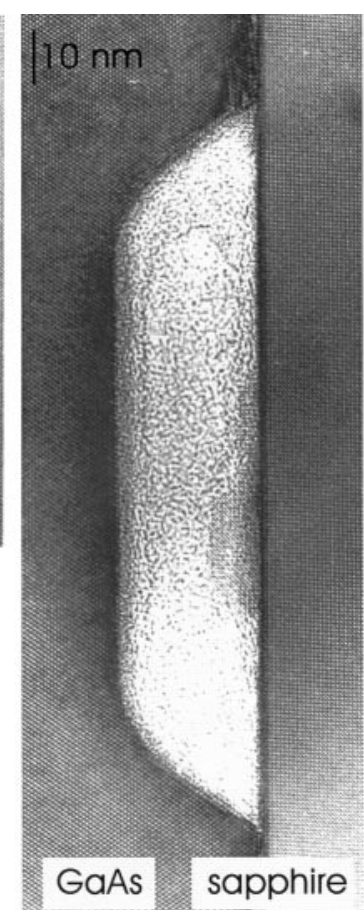




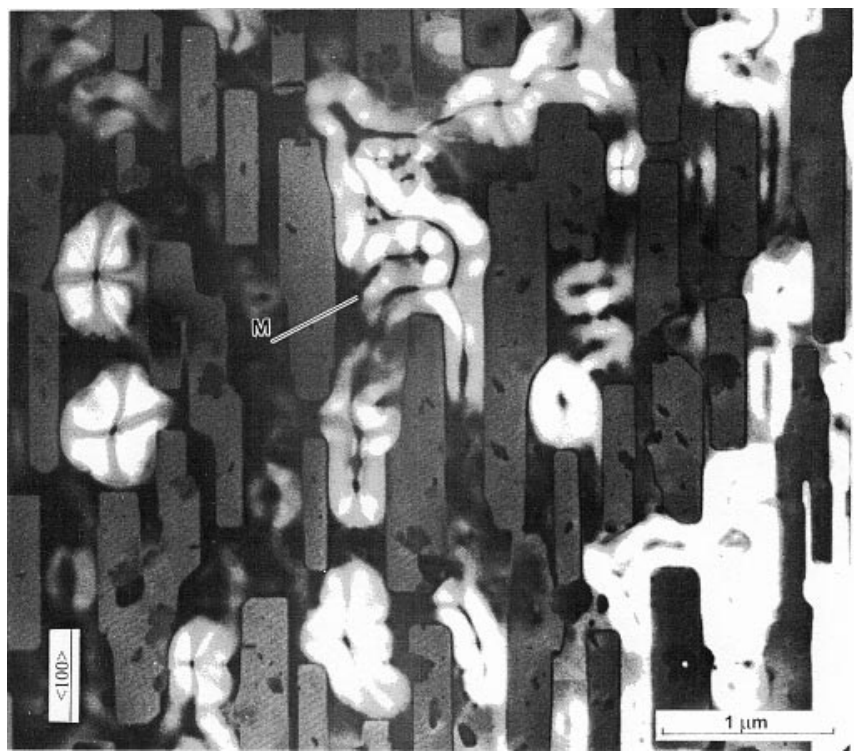

Fig. 8. Plan TEM view (transmission perpendicular to the interface plane) showing a rarely observed distribution of bonded and unbonded areas

with uniform, grey contrast elongated along a $\langle 100\rangle$ direction of the GaAs. The anisotropy of the pattern probably results form the anisotropy of the adjacent (1102) bond plane of sapphire. Within these areas, weak Moiré fringes are seen parallel to the direction indicated by $\mathrm{M}$. The bonded areas are separated by channel-like regions where diffraction contrast reveals prominent, strong bending contours. The latter clearly appear if the specimen is transmitted along the [100] direction of the GaAs as in the present case. The contours reveal a fourfold symmetry at their dark cross points where the local orientation is exactly [100] along the transmitting beam. This analysis indicates bending of the GaAs lattice that is locally bell-shaped but cylindrically elongated along unbonded "channels". Apparently such channels cannot open further to form an extended void since the bonded regions gained a relatively high bonding energy.

Bubble-free wafer bonding of gallium arsenide-onsapphire is achieved by bonding and annealing the wafers in a hydrogen atmosphere. The GaAs wafers are first bonded to sapphire hydrophilically as presented above. Prior to the annealing in hydrogen the wafer pairs are debonded by introducing small blades along the bonding interface. After removing the blades the wafers remain in contact but nevertheless they do not bond spontaneously by Van der Waals forces. The debonding of the wafer pair is necessary to allow the diffusion of hydrogen into the interface. The GOS wafer pairs are annealed for $5-6$ hours in a hydrogen flow of $8 \ell / \mathrm{min}$ at $500{ }^{\circ} \mathrm{C}$ without external pressure being applied. The high partial pressure of water in the hydrogen atmosphere means that the residual water monolayers evaporate from the wafer surface. Thus the chemical properties of the hydrophilic surfaces of the GaAs wafers are changed into those of a hydrophobic surface. At a specific temperature the nucleation of bonding between the GaAs and sapphire wafers starts spontaneously. The nucleation temperature is easily determined from the temperature-dependent bending of the GOS wafer pair shown in Fig. 3. The bending of a wafer pair is measured as $0.39 \mathrm{~m}^{-1}$ by profilometry. This corresponds to a temperature of bonding nucleation between $370{ }^{\circ} \mathrm{C}$ and $380^{\circ} \mathrm{C}$.

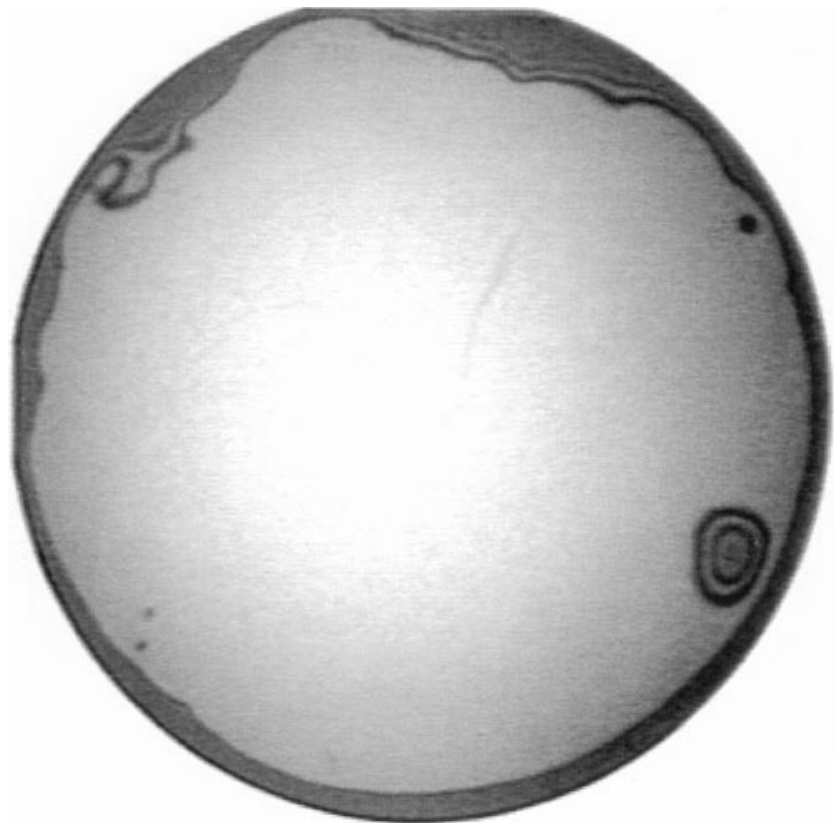

Fig. 9. Infrared transmission picture of a GOS wafer pair, bonded in a hydrogen atmosphere and annealed at $500{ }^{\circ} \mathrm{C}$ for 6 hours. Only very few small bubbles are observed

The ultimate bonding energy is comparable to the bonding energy of GaAs bulk material. Figure 9 shows an infrared transmission picture of the annealed GOS wafer pair. Only very few small bubbles are observed. Formation of large interface bubbles, typical for hydrophilic bonding, is prevented by this approach. Closer inspection by optical microscopy does not reveal any additional bubbles.

\section{Summary and outlook}

Direct wafer bonding of 3-inck. GaAs wafers to sapphire wafers (GOS) is investigated. After annealing at $500^{\circ} \mathrm{C}$ the bonding energy is comparable to the energy of covalent bonding in the bulk GaAs. The bonded wafer pair is mechanically stable during heating or cooling to liquid nitrogen temperatures. Interface bubbles developed at fairly low temperatures if the wafers are bonded under hydrophilical surface conditions. TEM cross sections of the bonded areas show a sharp GaAs/sapphire interface. Planar TEM specimens revealed small inhomogeneities, corresponding to locally unbonded areas. In particular, grooves filled with amorphous material are found along the interface. We suppose that a network of micro-channels provides the mass transport to form the macro-bubbles.

By changing the chemical properties of the hydrophilical surfaces to hydrophobic by bonding and annealing in a hydrogen atmosphere, we avoid the growth of bubbles at the GaAs/sapphire interface. Because of the low thermal strain and the high bond energy achieved, the direct bonding of GaAs to sapphire offers new possibilities for fabricating hybrid electronic devices, e.g. gallium arsenide electronic devices combined with microwave amplifiers or filters based on high-temperature superconducting films epitaxially grown on sapphire. 
Acknowledgements. Thanks are due to Dr.N. Zakharov for taking TEM crosssectional lattice images. This work was partly supported by the German Ministry of Research (BMBF) under contracts 13N6451 and 13N6758.

\section{References}

1. H. Baumgart, C. Hunt, M. Schmidt, T. Abe (eds.): Proc. 2 rd Int. Symp. Semiconductor Wafer Bonding: Science, Technology and Applications II (Electrochem. Soc., Pennington, NJ 1993)

2. C. Hunt, H. Baumgart, S. Iyer, U. Gösele, T. Abe (eds.): Proc. 3rd Int. Symp. Semiconductor Wafer Bonding: Science, Technology and Applications (Electrochem. Soc. , Pennington, NJ 1995)

3. S. Bengtsson: J. Electronic Materials 21, 669 (1992)

4. Special Issue on Direct Bonding, Philips J. Res. 49 (1995)

5. U. Gösele, H. Stenzel, M. Reiche, T. Martini, H. Steinkirchner, Q.Y. Tong: Solid State Phenomena 47/48, 33 (1996)

6. E. Yablonovitch, D.M. Hwang, T.J. Gmitter, L.T. Florez, J.P. Harbison: Appl. Phys. Lett. 56, 2419 (1990)
7. J. Haisma, G.A.C.M. Spierings, U.K.P. Biermann, A.A.van Gorkum: Appl. Opt. 33, 1154 (1994)

8. T. Takamori, H. Wada, T. Kamijoh: Appl. Surf. Sci. in press

9. T. Sugino, T. Itagaki, J. Shirafuji: Diamond and Related Materials 5, 714 (1996)

10. L. Di Cioccio, Y. Le Tiec, F. Letertre, C. Jaussaud, M. Bruel: Electron. Lett. Vol. 32, 1144 (1996)

11. Z.-Y. Shen: In High-Temperature Superconducting Microwave Circuits, (Artech House, Inc., London 1994), p.193

12. J.M. Phillips: J. Appl. Phys. 79, 1829 (1996)

13. T. Stengl, K.-Y. Ahn, U. Gösele: Jpn. J. Appl. Phys. 27, 2364 (1988)

14. W.P. Maszara, G. Goertz, A. Cavilia, J.B. McKnitterick: J. Appl. Phys. 64, 4943 (1988)

15. K. Mitani, V. Lehmann, R. Stengl, D. Feijoo, U.M. Gösele, H.Z. Massoud: Jpn. J. Appl. Phys. 30, 615 (1991)

16. P. Kopperschmidt, St. Senz, G. Kästner, D. Hesse, U.M. Gösele: Proc. 23th Int. Conf Phys. Semiconductors (ICPS), Berlin (1996), ed. by M. Scheffler and R. Zimmermann, (World Scientific, Singapore 1996), p.967

17. H.L. Hartnagel: In Properties of Gallium Arsenide, 2nd ed. (INSPEC, London 1990), p.369 